\title{
Modifications in Interaction and Structure of Silica Nanoparticle-BSA Protein System in Aqueous Electrolyte Solution
}

\author{
Indresh Yadav ${ }^{1}$, V.K. Aswal ${ }^{1, *}$ and J. Kohlbrecher ${ }^{2}$ \\ ${ }^{1}$ Solid State Physics Division, Bhabha Atomic Research Centre, Mumbai-400085, India \\ ${ }^{2}$ Laboratory for Neutron Scattering, Paul Scherrer Institut, CH-5232 PSI Villigen, Switzerland
}

*E-mail:vkaswal@barc.gov.in

\begin{abstract}
SANS measurements have been carried out to examine the modifications in interaction and structure of anionic silica nanoparticle with anionic BSA protein in presence of an electrolyte. The phase behaviour of anionic silica nanoparticle and anionic BSA protein is governed by the protein induced depletion interaction between nanoparticles. Both nanoparticle and protein coexist individually at low protein concentrations as electrostatic repulsion dominates over the depletion interaction. However, depletion induced fractal aggregates of nanoparticles are formed at higher protein concentrations. These aggregates can be formed at much smaller protein concentration in presence of an electrolyte. We show that both the electrostatic (decrease) and depletion interaction (increase) are modified with an electrolyte. The range of the depletion interaction is found to be significantly larger than the electrostatic interaction.
\end{abstract}

Keywords: Colloids, nanoparticle-protein interaction, small-angle neutron scattering

PACS: 82.70.Dd, 61.46.-w, 61.05.fg

\section{INTRODUCTION}

Colloidal dispersions of nanoparticles and proteins show rich phase behaviour by providing a model system to study the interaction of nanoparticles with biomolecules [1]. The intrinsic characteristic of nanoparticle (e.g. size, shape and charge) and the forces (e.g. electrostatic, hydrogen bonding and steric forces) at the bio-nano interface control the phase behaviour, which can be tuned by varying different solution parameters such as ionic strength, $\mathrm{pH}$, temperature and concentration. There have been a number of studies of the interaction of silica nanoparticles with both the similarly and oppositely charged proteins. In the case of oppositely charged protein, the adsorption of protein on nanoparticles leads to the protein mediated aggregation of nanoparticles [2]. On the other hand, for similarly charged protein, site-specific adsorption of protein on nanoparticles has been reported [3]. We have recently reported that nanoparticle aggregation can also be observed in similarly charged protein, which is identical to that with the oppositely charged protein [4]. The non-adsorbing nature of similarly charged protein induces the depletion force that causes the aggregation of nanoparticles at higher protein concentrations. Herein, we have looked into the role of addition of an electrolyte in the interaction and structure of such nanoparticle-protein systems. These systems have been characterized by small-angle neutron scattering (SANS). The technique of SANS gives the scattering patterns in Fourier space form where the real space sample information is obtained by fitting experimental data using a suitable model. Both the structure and interaction can be obtained using this method in in-situ and under native environment.

\section{EXPERIMENT}

Silica nanoparticles (Ludox HS-40) and bovine serum albumin (BSA) proteins were purchased from Sigma-Aldrich. Samples were prepared by dissolving the weighted amount of nanoparticles and proteins in $20 \mathrm{mM}$ phosphate buffer at $\mathrm{pH} \mathrm{7,} \mathrm{without} \mathrm{and} \mathrm{with}$ $0.1 \mathrm{M} \mathrm{NaCl}$, and prepared in $\mathrm{D}_{2} \mathrm{O}$. Samples for neutron scattering experiments are prepared in $\mathrm{D}_{2} \mathrm{O}$ instead of $\mathrm{H}_{2} \mathrm{O}$ because of high contrast for hydrogenous sample in $\mathrm{D}_{2} \mathrm{O}$. Small-angle neutron scattering experiments were performed at the SANS-I instrument at the Swiss spallation neutron source, SINQ, Paul Scherrer Institut, Switzerland [5]. The data were collected to in scattering vector $(Q=4 \pi \sin (\theta / 2) / \lambda$, where $\theta$ is 
scattering angle) range of $0.006-0.25 \AA^{-1}$. All the measurements were carried out for fixed concentration (1 wt $\%)$ of silica nanoparticles and varying the concentration of protein in the range 0 to $5 \mathrm{wt} \%$. The freshly prepared samples were held in HELLMA quartz cells having thickness $2 \mathrm{~mm}$ and temperature kept constant at $30{ }^{\circ} \mathrm{C}$ during the measurements. The data were corrected and normalized to absolute scale using standard procedure.

\section{SANS ANALYSIS}

In SANS experiment, the coherent differential scattering cross-section per unit volume $(d \Sigma / d \Omega)$ is measured as given by

$$
\frac{d \Sigma}{d \Omega}(Q)=n V^{2}\left(\rho_{p}-\rho_{s}\right)^{2} P(Q) S(Q)+B
$$

where $n$ is number density and $V$ is particle volume. $\rho_{\mathrm{p}}$ and $\rho_{\mathrm{s}}$ are scattering length densities of particles and solvent, respectively. $P(Q)$ is intraparticle structure factor and $S(Q)$ is interparticle structure factor. B is a constant term representing incoherent background.

$P(Q)$ depends on the geometry of the particle whereas $S(Q)$ correlates particles present in the system and gives information about the interactions in the system. The evolution of interaction between nanoparticles on addition of protein has been calculated using two Yukawa potential under mean spherical approximation [6]. The two-Yukawa potential having 4 dimensionless parameters $\left(K_{1}, K_{2}\right.$, $Z_{1}$ and $\left.Z_{2}\right)$ is expressed by

$$
\begin{aligned}
\frac{V(r)}{k_{B} T} & =\infty \text { for } 0<r<\sigma \\
& =-K_{1} \frac{\exp \left\{-Z_{1}(r / \sigma-1)\right\}}{r / \sigma}+K_{2} \frac{\exp \left\{-Z_{2}(r / \sigma-1)\right\}}{r / \sigma} \text { for } r>\sigma
\end{aligned}
$$

where $\sigma$ is hard sphere diameter of the nanoparticle.

In the case of particle aggregation as characterized by fractal structure, the structure factor $S(Q)$ for mass fractal is given by

$$
S(Q)=1+\frac{1}{(Q R)^{D}} \frac{D \Gamma(D-1)}{\left[1+\frac{1}{(Q \xi)^{2}}\right]^{(D-1) / 2}} \sin \left\{(D-1) \times \tan ^{-1}(Q \xi)\right\}
$$

where $\xi$ signifies the maximum length up to which fractal microstructure exists, $R$ is the size of building block and $D$ is the fractal dimension. $\Gamma$ is the mathematical gamma function.

\section{RESULTS AND DISCUSSION}

SANS data of $1 \mathrm{wt} \%$ silica nanoparticle with varying BSA protein concentration in presence of an electrolyte $(0.1 \mathrm{M} \mathrm{NaCl})$ are shown in Figure 1. The data show a systematic build-up of scattering in the low and high $Q$ regions as the protein concentration is increased. The build-up of scattering in the low $Q$ region arises because of the increase in attractive interaction between nanoparticles. The increase in high $Q$ scattering results from the increase in protein concentration. Both these features at low and high $Q$ values can be explained on the basis that non-adsorption of BSA protein gives rise to the attractive depletion interaction whose magnitude increases with the increase in protein concentration. The scattering from the non-adsorbed (free) protein reflects in the high $Q$ region since its size is much smaller than that of the nanoparticle. The low $Q$ scattering at high protein concentration tends to be linear on log-log scale, indicating formation of protein depletion induced fractal aggregates of nanoparticles [4]. The amount of protein concentration required for inducing nanoparticle aggregates is significantly reduced (about an order) in presence of an electrolyte $(0.1 \mathrm{M} \mathrm{NaCl})$. The inset of Figure 1 compares the SANS data of silica nanoparticle-BSA protein system without and with an electrolyte. It is clear that the scattering intensity in the low $Q$ region has been significantly enhanced in presence of an electrolyte.

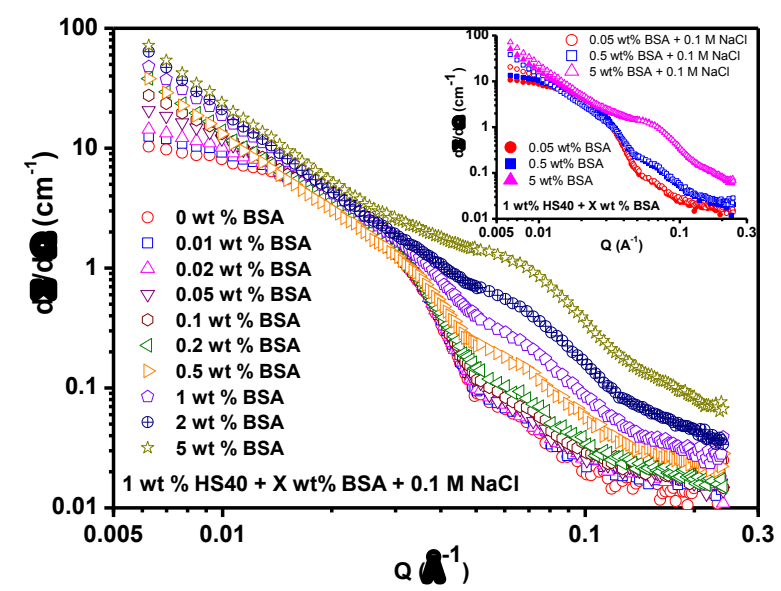

FIGURE 1. SANS data of $1 \mathrm{wt} \%$ HS40 silica nanoparticles with varying concentration of BSA protein in presence of $0.1 \mathrm{M} \mathrm{NaCl}$. Inset shows selective comparison of SANS data with and without an electrolyte.

Figure 2 shows the fitted SANS data of $1 \mathrm{wt} \%$ $\mathrm{HS} 40+0.5 \mathrm{wt} \%$ BSA without and with $0.1 \mathrm{M} \mathrm{NaCl}$. A polydisperse spherical model is used to fit the scattering profile of pure silica nanoparticles. The mean radius of silica nanoparticles is found $88.0 \AA$ with polydispersity 0.2 . On the other hand, BSA has an oblate ellipsoidal shape with semimajor and semiminor axes $42.0 \AA$ and $15.0 \AA$, respectively. The inset of figure shows that differences in $S(Q)$ of the two systems. Data are fitted using a two-Yukawa potential accounting for both repulsive and attractive parts of total interaction potential between nanoparticles. 
The potential comprises 4 fitting parameters $\left(K_{1}, K_{2}\right.$, $Z_{1}, Z_{2}$ ), where $K_{1}$ and $Z_{1}$ are the fitting parameters of the attractive potential to give the strength (proportional to $K_{1}$ ) and range (proportional to $1 / Z_{1}$ ), respectively [6]. $\quad K_{2}$ and $Z_{2}$ are the fitting parameters of the repulsive potential which provide the strength (related to effective charge) and range (related to ionic strength), respectively. The parameters of repulsive potential have been determined from the extrapolation of pure concentrated solution of nanoparticles as it is difficult to see the $S(Q)$ contribution from repulsive interaction in $1 \mathrm{wt} \%$ or lower concentration of nanoparticle system. All the fitted parameters are given in Table 1.

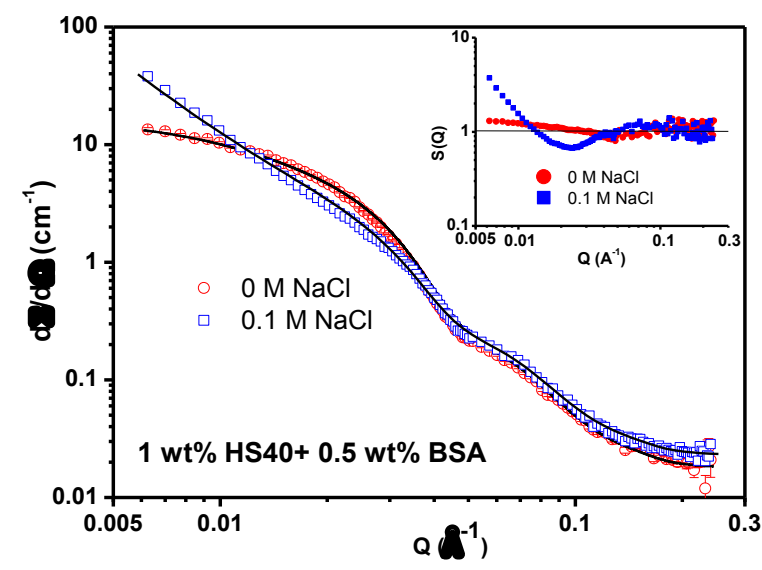

FIGURE 2. Fitted SANS data of $1 \mathrm{wt} \%$ HS40 + $0.5 \mathrm{wt} \%$ BSA without and with an electrolyte $(0.1 \mathrm{M} \mathrm{NaCl})$. Inset show the $S(Q)$ in these two systems.

TABLE 1. The parameters of two-Yukawa potential in fitting SANS data of $1 \mathrm{wt} \% \mathrm{HS} 40+0.5 \mathrm{wt} \%$ BSA without and with $0.1 \mathrm{M} \mathrm{NaCl}$.

\begin{tabular}{ccccc}
\hline [Salt] & $K_{1}$ & $Z_{1}$ & $K_{2}$ & $Z_{2}$ \\
\hline $0 \mathrm{M} \mathrm{NaCl}$ & 10.5 & 4.0 & 9.0 & 7.0 \\
$0.1 \mathrm{M} \mathrm{NaCl}$ & 35.0 & 4.0 & 1.5 & 19.5 \\
\hline
\end{tabular}

The depletion interaction in Table 1 is found to be long-range (range $=1 / Z_{1}$ ) as is the case of electrostatic interaction [7]. The parameters of electrostatic interaction in presence of an electrolyte vary in accordance with the corresponding change in Debye length. This results in decrease in value of magnitude $\left(K_{2}\right)$ and range $\left(1 / Z_{2}\right)$ of the repulsive interaction. On the other hand, for depletion interaction, the magnitude $\left(K_{1}\right)$ increases whereas range $\left(1 / Z_{1}\right)$ remains same. The increase in magnitude of depletion interaction can be understood in terms of decrease in protein-protein repulsion in presence of an electrolyte. The suppression of repulsion significantly increases the excluded volume available for proteins and hence higher magnitude of depletion interaction. The total potential between nanoparticles along with its electrostatic and depletion components are plotted in figure 3. These results thus show that presence of an electrolyte leads in significant modifications in both electrostatic and depletion interactions. The value of fractal dimension of the nanoparticle aggregation at higher protein concentration is found to be around 2.5, suggesting it is governed by the process of diffusion limited aggregation (DLA) [2].

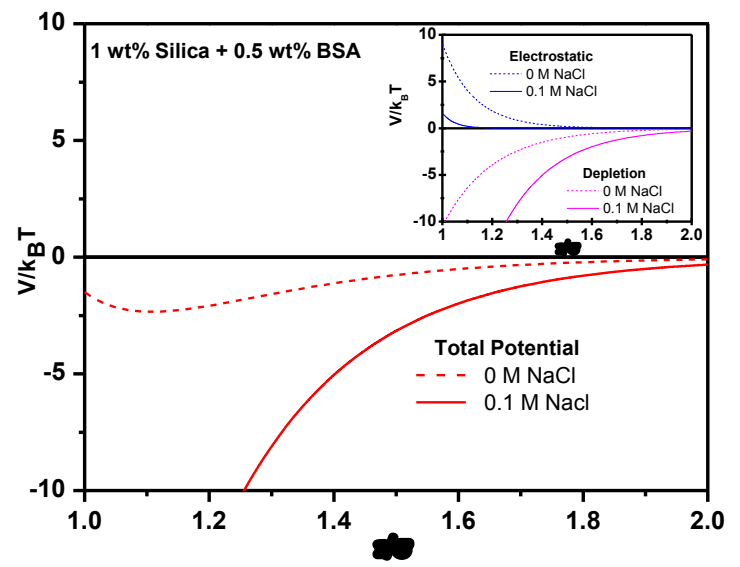

FIGURE 3. The calculated total interaction potential between nanoparticles in $1 \mathrm{wt} \%$ HS40 +0.5 wt $\%$ BSA without and with $0.1 \mathrm{M} \mathrm{NaCl}$. Inset shows the electrostatic and depletion components of the total potential.

\section{CONCLUSION}

The interplay of electrostatic and depletion interactions decide the phase behaviour of nanoparticle with non-adsorbing protein. The electrostatic interaction as expected is suppressed whereas depletion interaction is enhanced in presence of an electrolyte. The modifications of these interactions together favour the aggregation of nanoparticles at much lower protein concentration. The aggregates are characterized by DLA type of mass fractal morphology.

\section{REFERENCES}

1. I. Lynch and K.A. Dawson, Nano Today 3, 40 (2008).

2. S. Kumar, V.K. Aswal and J. Kohlbrecher, Langmuir 27, 10167-10173 (2011).

3. A. M. Yake, C. E. Snyder and D. Velegol, Langmuir 23, 9069-9075 (2007).

4. I. Yadav, S. Kumar, V.K. Aswal and J. Kohlbrecher, Phy. Rev. E 89, 032304 (2014)

5. J. Kohlbrecher and W. Wagner, J. Appl. Crystallogr. 33, 804-806 (2000).

6. Y. Liu, W.-R. Chen and S.-H. Chen, J. Chem. Phys. 122, 044507 (2005).

7. S.Kumar, M.-J. Lee, V. K. Aswal and S.-M. Choi. Phys. Rev. E 87, 042315 (2013). 